Supplemental Materials

\title{
Linalool derivatives for natural product-based 4D printing resins
}

David Merckle ${ }^{1}$, Eric Constant ${ }^{2}$, Andrew C. Weems ${ }^{1-3^{*}}$

1. Translational Biosciences, Ohio University, Athens, $\mathrm{OH}, 45701$

2. Biomedical Engineering Program, Ohio University, Athens, OH, 45701

3. Department of Mechanical Engineering; Molecular and Chemical Biology; Orthopedic Musculoskeletal and Neurological Institute, Ohio University, Athens, OH, 45701

*corresponding author's email address: weemsac@ohio.edu

Number of Pages

Number of Figures

Number of Tables

\section{Table of Contents}

$\begin{array}{ll}\text { Spectroscopic Characterization } & \text { S2 }\end{array}$

$\begin{array}{ll}\text { Rheological Characterization } & \mathrm{S} 12\end{array}$

$\begin{array}{ll}\text { Photorheology } & \text { S13 }\end{array}$

UV-Visible Light Photospectroscopy $\quad$ S14

$\begin{array}{ll}\text { Thermogravimetric Analysis } & \text { S15 }\end{array}$

$\begin{array}{ll}\text { Dynamic Mechanical Analysis } & \text { S16 }\end{array}$

$\begin{array}{ll}\text { Tensile Mechanical Properties (Table) } & \text { S17 }\end{array}$

$\begin{array}{ll}\text { Micro CT } & \text { S18 }\end{array}$

4D/Shape Memory Behavior $\quad$ S21 
Spectroscopic Characterization

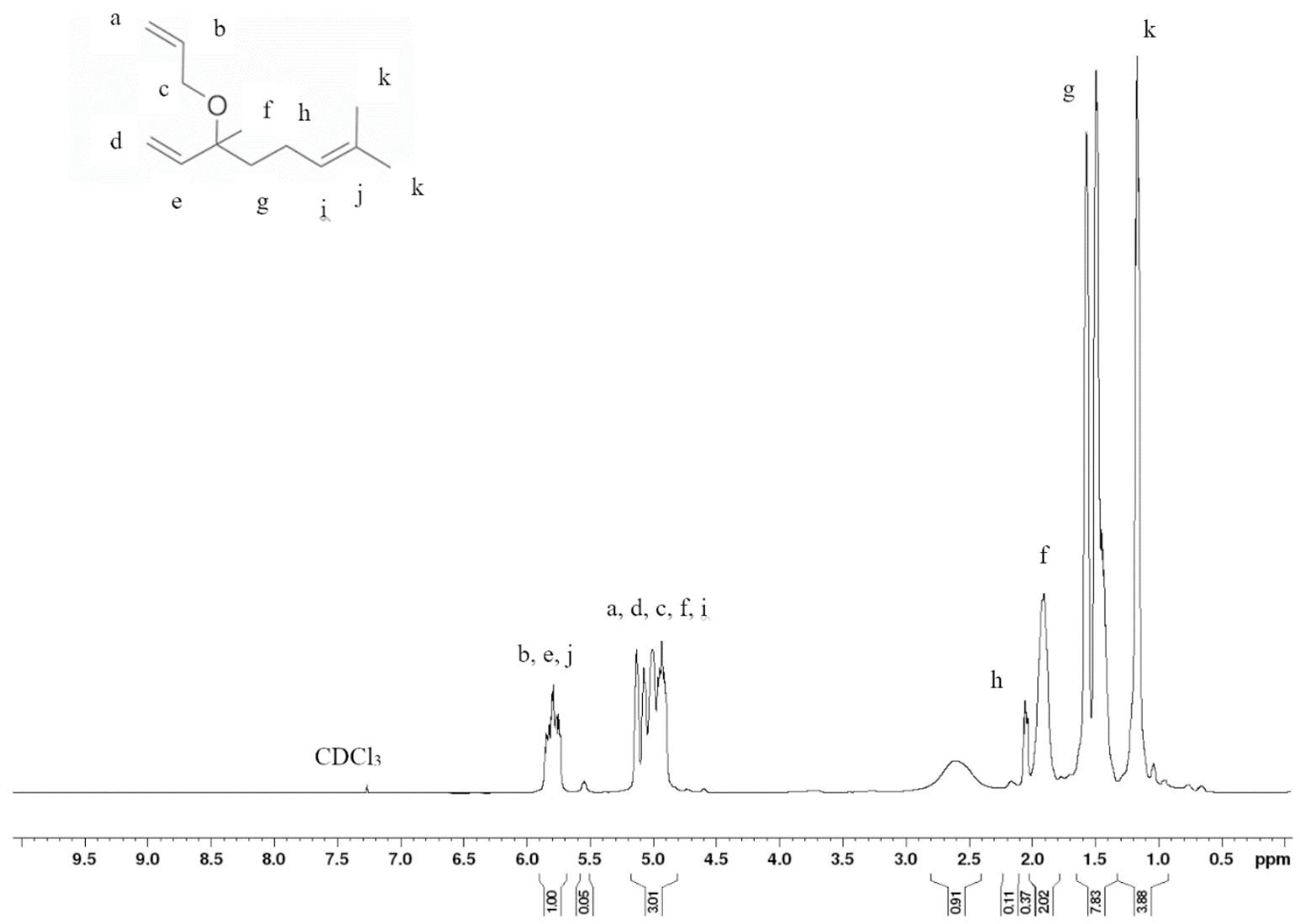

Figure S1. ${ }^{1} \mathrm{H}$ NMR of allyl linalool monomer in $\mathrm{CDCl}_{3}(298 \mathrm{~K}, 500 \mathrm{MHz})$. 


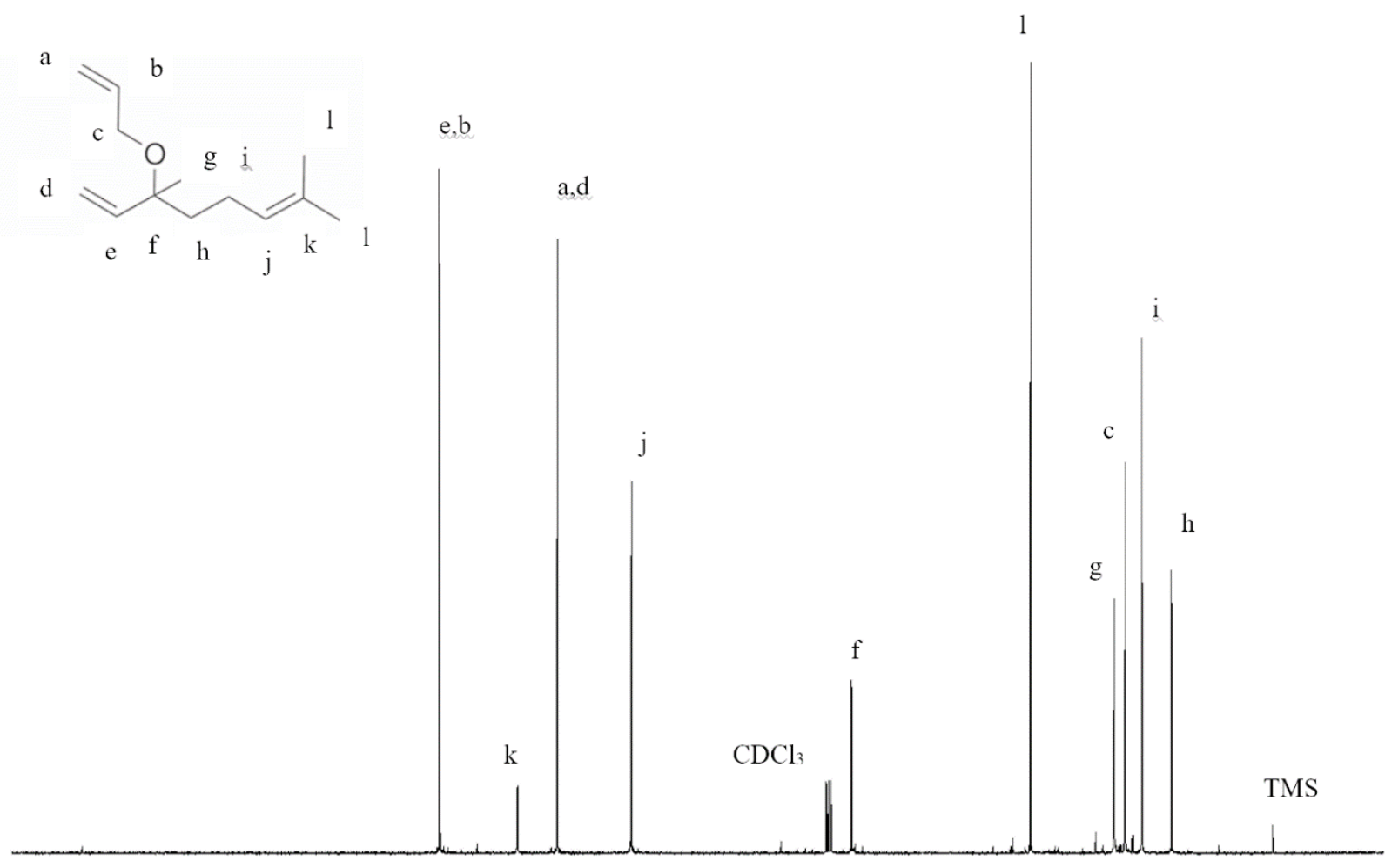

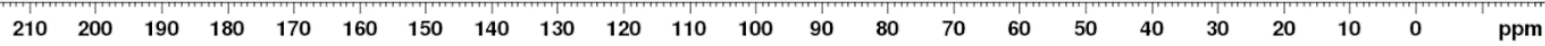
Figure S2. ${ }^{13} \mathrm{C}$ NMR of allyl linalool monomer in $\mathrm{CDCl}_{3}(298 \mathrm{~K}, 125 \mathrm{MHz})$. 


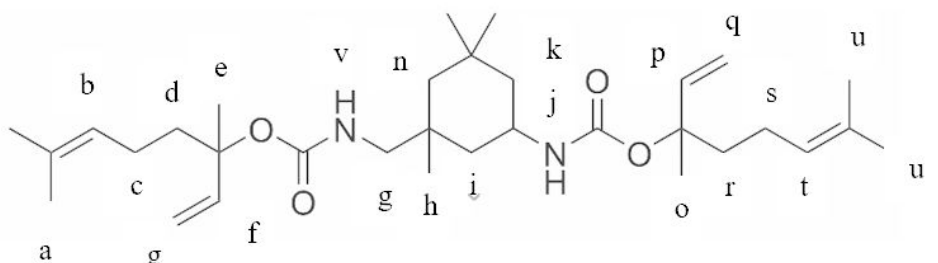

a

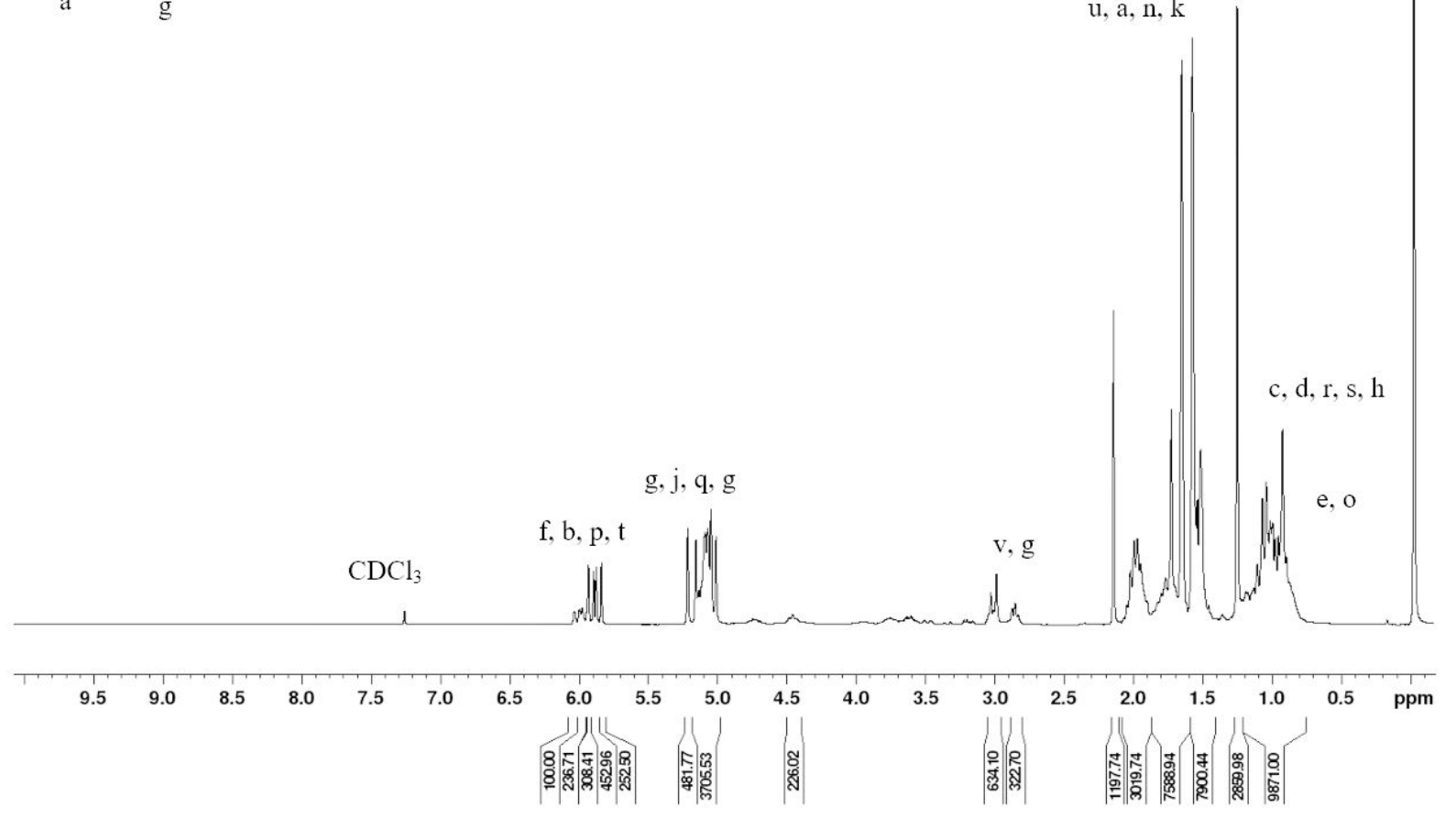

Figure S3. ${ }^{1} \mathrm{H}$ NMR of IPDI linalool monomer in $\mathrm{CDCl}_{3}(298 \mathrm{~K}, 500 \mathrm{MHz})$. 


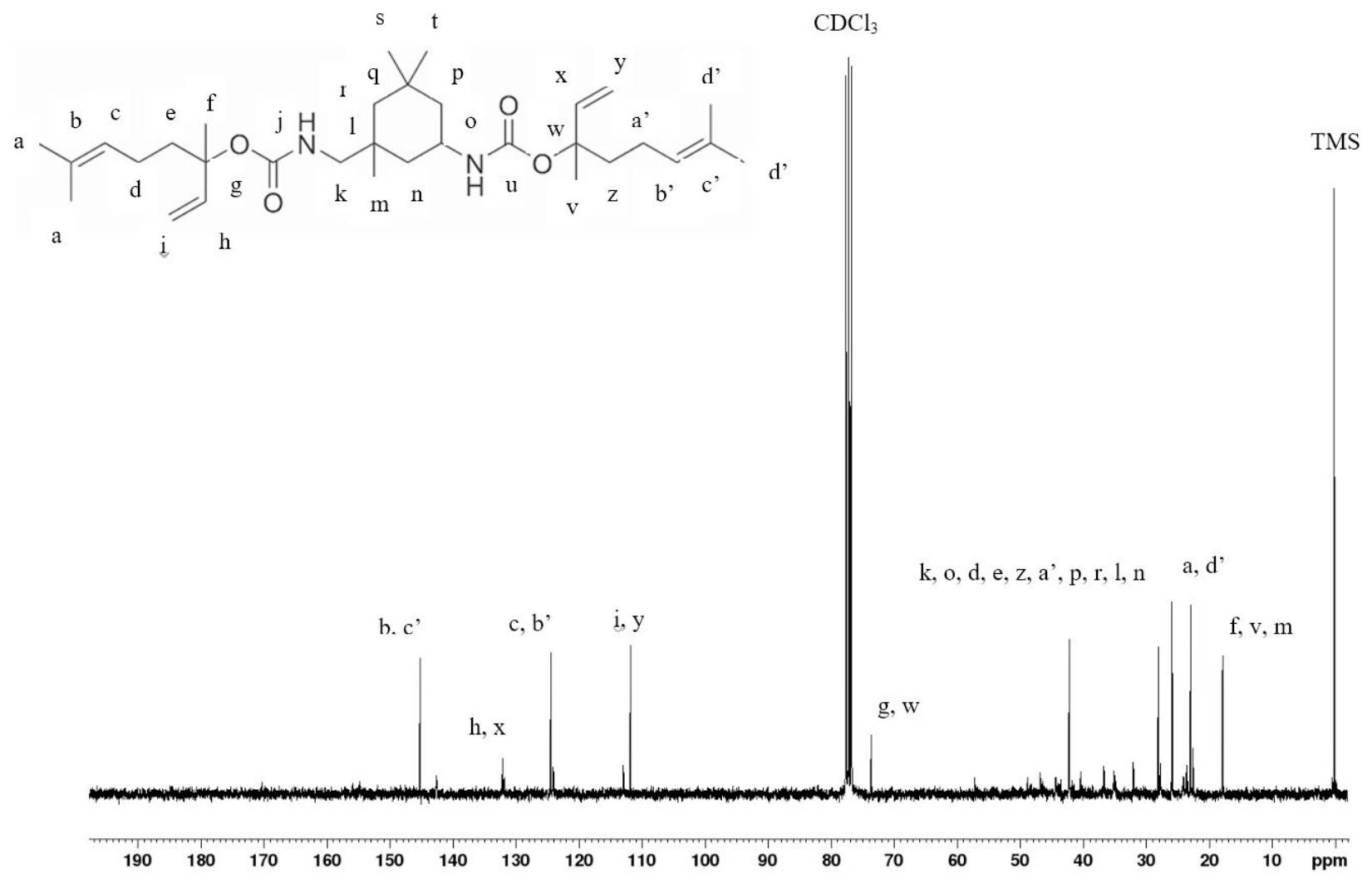

Figure S4. ${ }^{13} \mathrm{C}$ NMR of IPDI linalool monomer in $\mathrm{CDCl}_{3}(298 \mathrm{~K}, 125 \mathrm{MHz})$. 
a
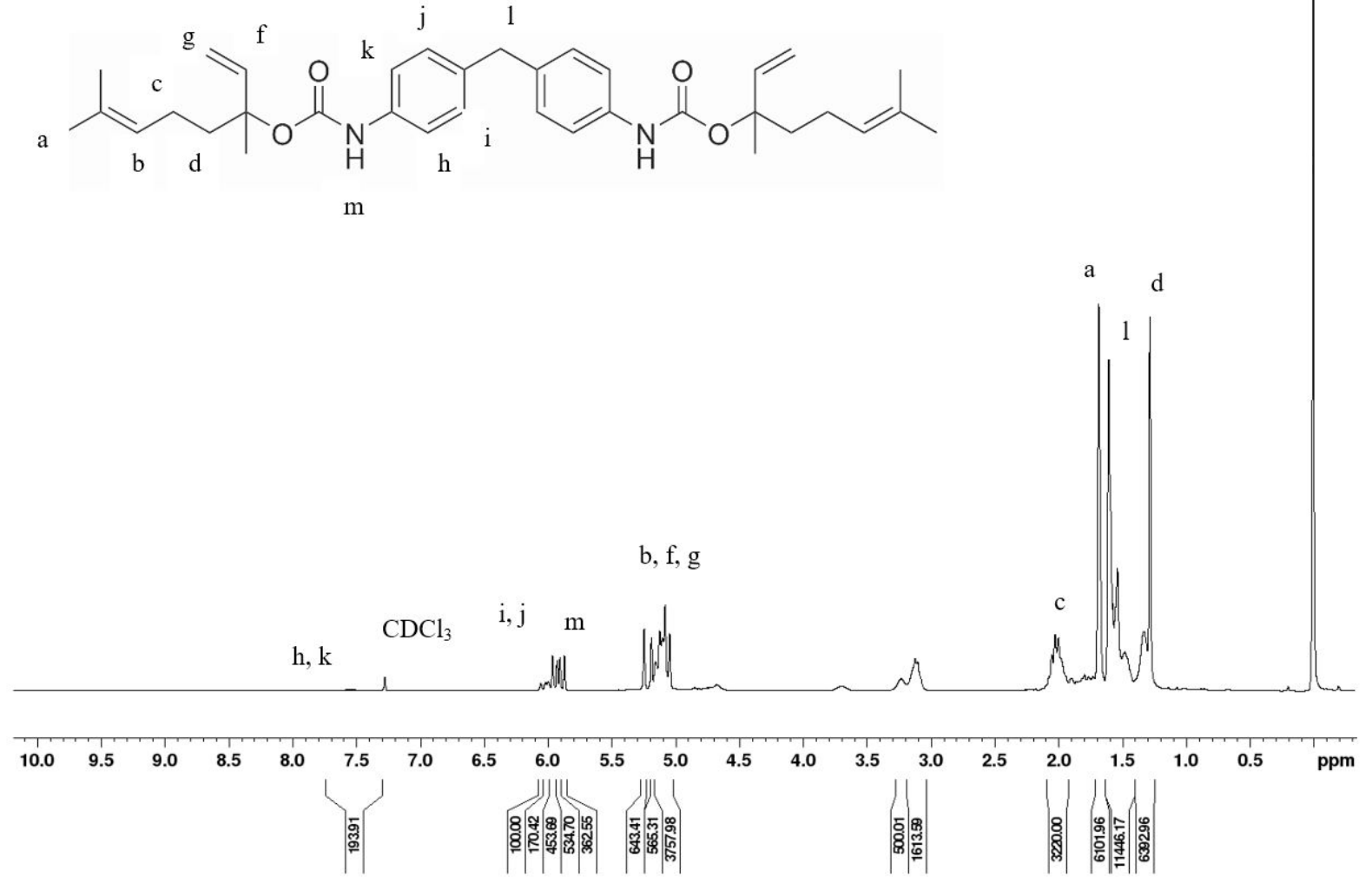

Figure S5. ${ }^{1} \mathrm{H}$ NMR of MDI linalool monomer in $\mathrm{CDCl}_{3}(298 \mathrm{~K}, 500 \mathrm{MHz})$. 


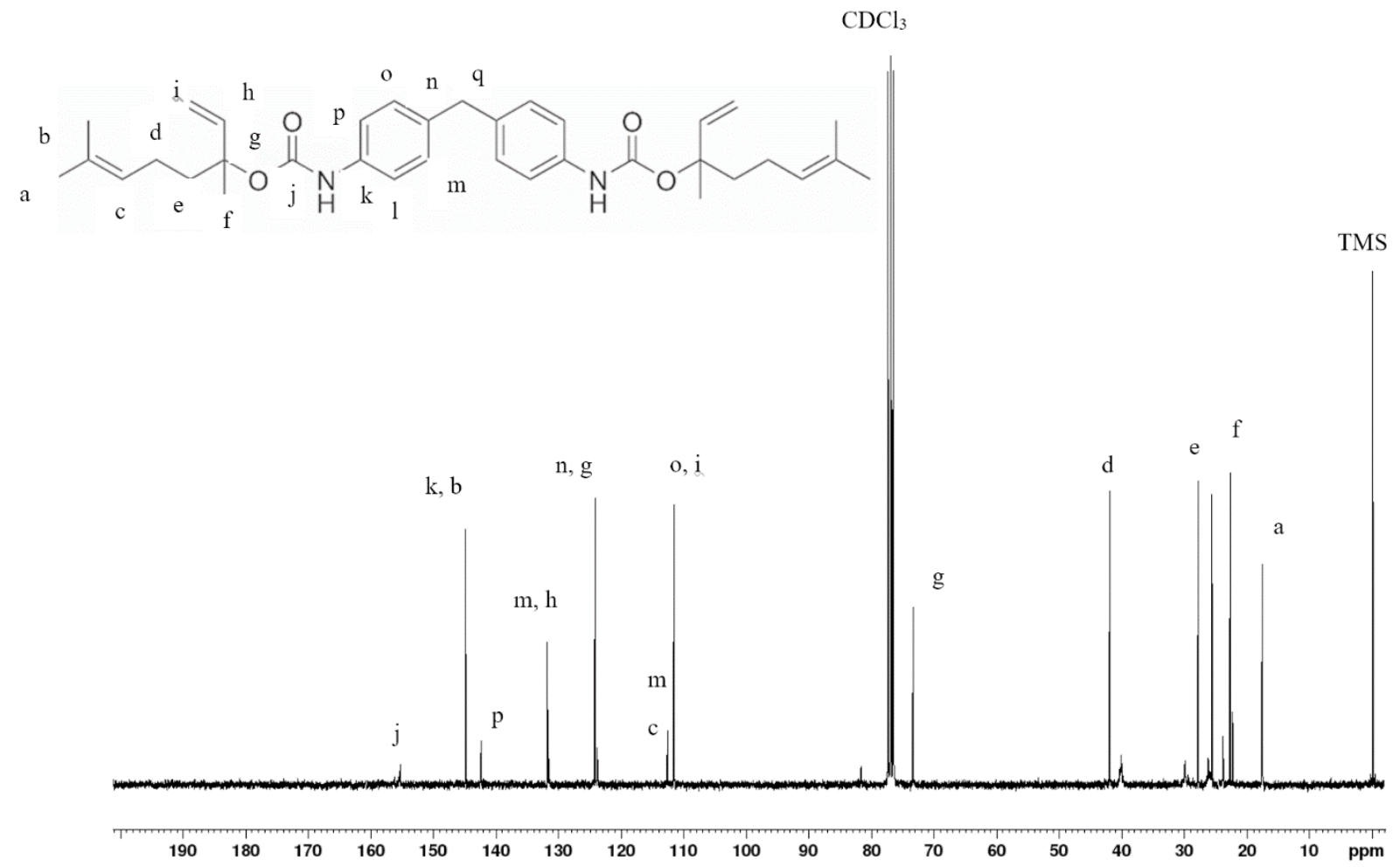

Figure S6. ${ }^{13} \mathrm{C}$ NMR of MDI linalool monomer in $\mathrm{CDCl}_{3}(298 \mathrm{~K}, 125 \mathrm{MHz})$. 


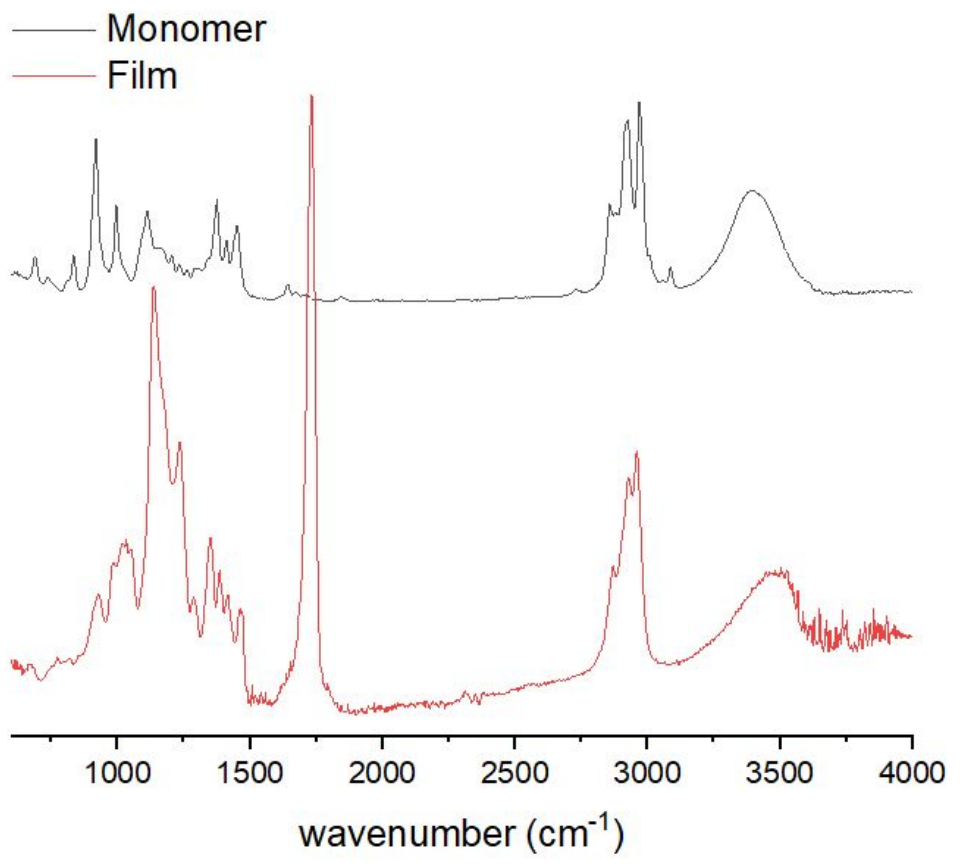

Figure S7. FT-IR of allyl linalool monomer and thermoset film after thiol-ene photocrosslinking. 


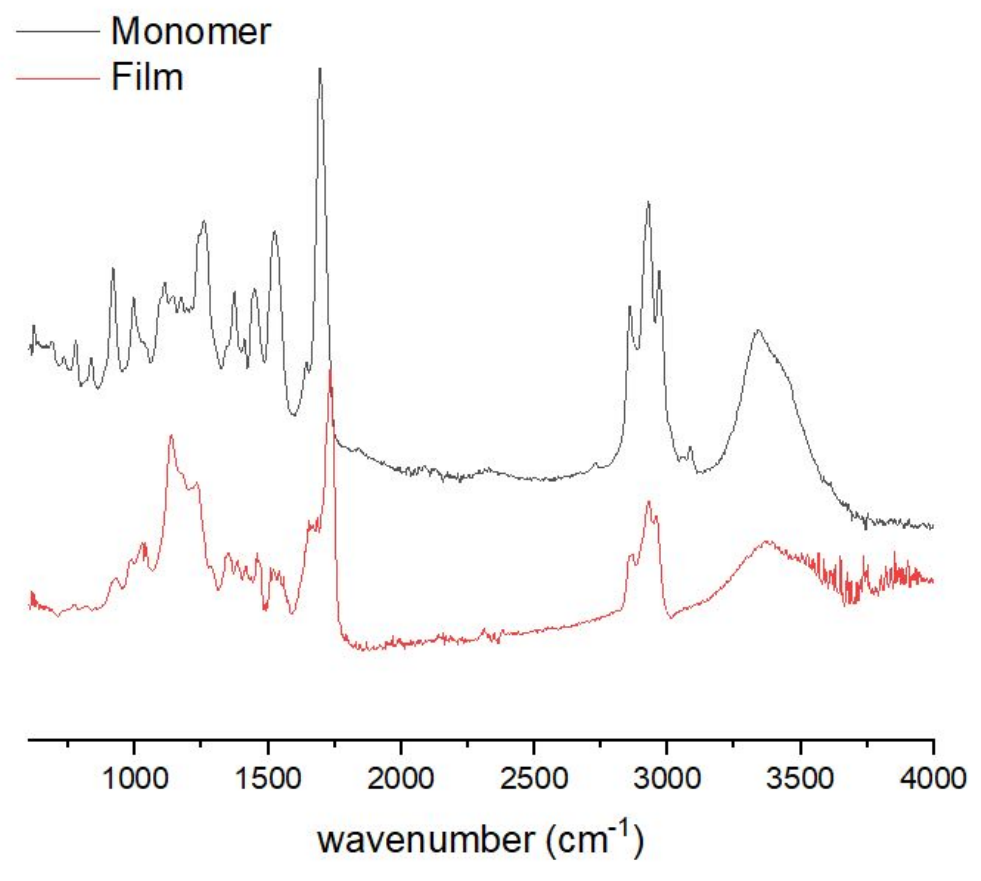

Figure S8. FT-IR of HDI linalool monomer and thermoset film after thiol-ene photocrosslinking. 


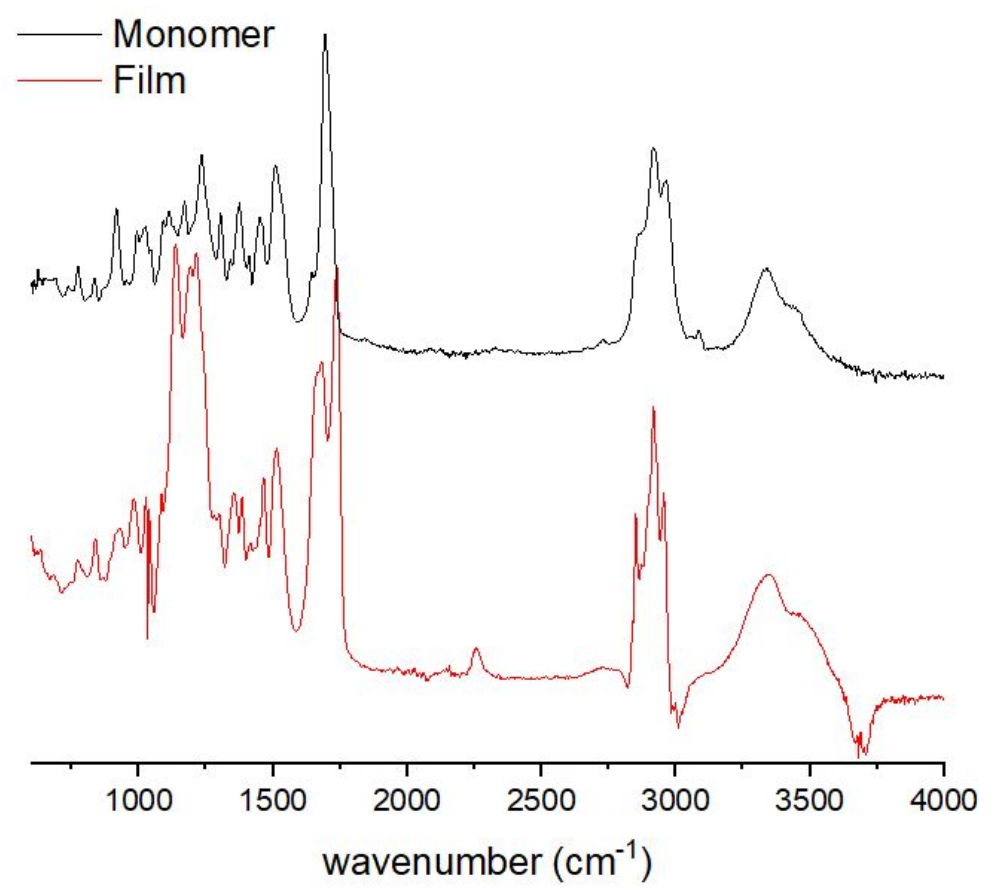

Figure S9. FT-IR of IPDI linalool monomer and thermoset film after thiol-ene photocrosslinking. 


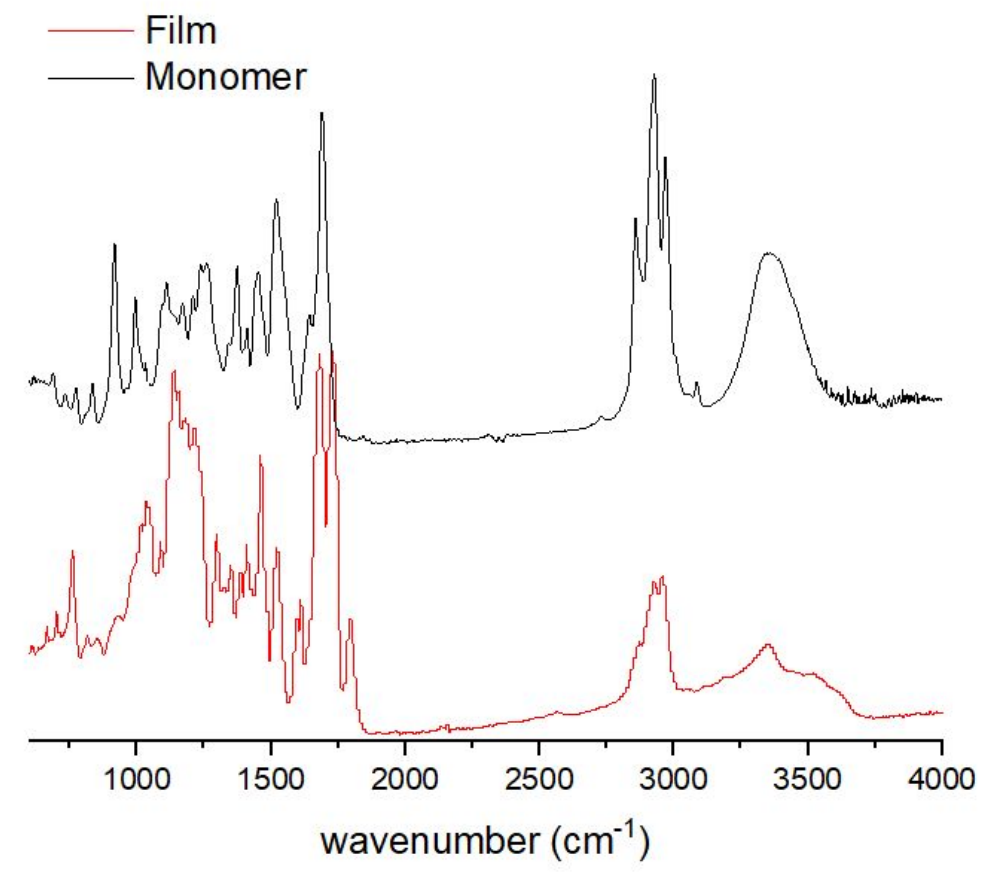

Figure S10. FT-IR of MDI linalool monomer and thermoset film after thiol-ene photocrosslinking. 
Rheological Characterization

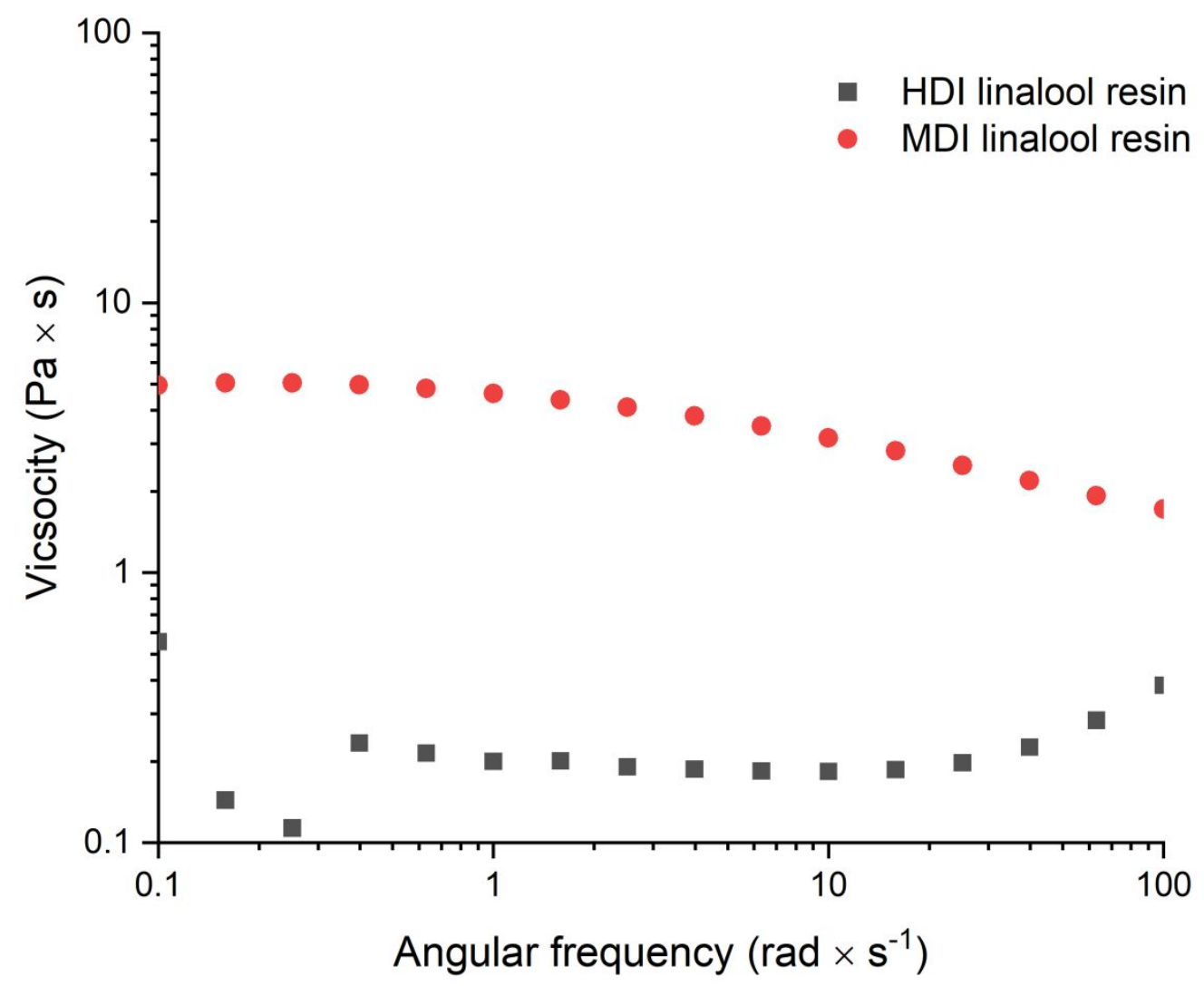

Figure S11. Representative angular frequency sweeps for parallel plate rheological analysis of HDI linalool and MDI linalool resin samples ( $40 \mathrm{~mm}$ stainless steel plate, $500 \mu \mathrm{m}$ gap, ambient conditions, 10 $\mathrm{Hz}$ frequency). 

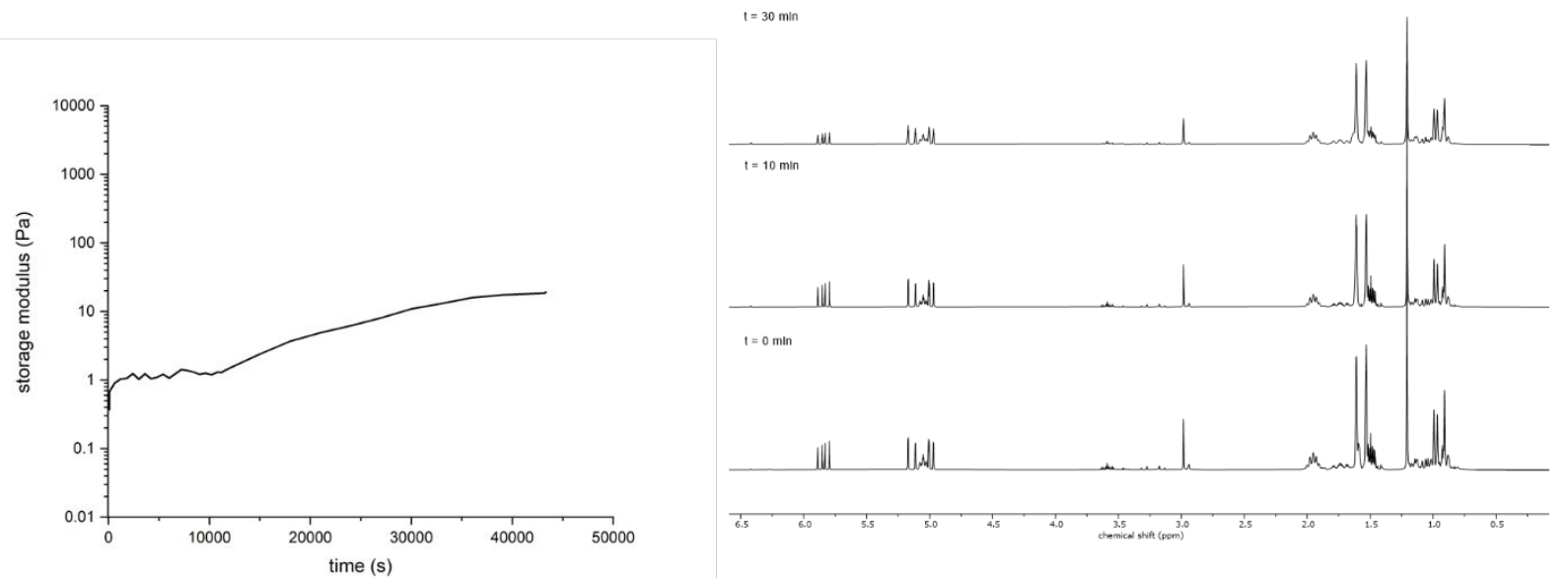

Figure S12. Photorheology of linalool monomer ( $0.5 \mathrm{wt} \%$ photoinitiator) during photoirradiation (365 to $750 \mathrm{~nm}$ light, $20 \mathrm{~W}$ ) (A) and ${ }^{1} \mathrm{H}$ NMR of the linalool IPDI (same conditions) irradiated for 0 , 10, and 20 minutes $\left(500 \mathrm{MHz}, \mathrm{CDCl}_{3}, 298 \mathrm{~K}\right)$. 


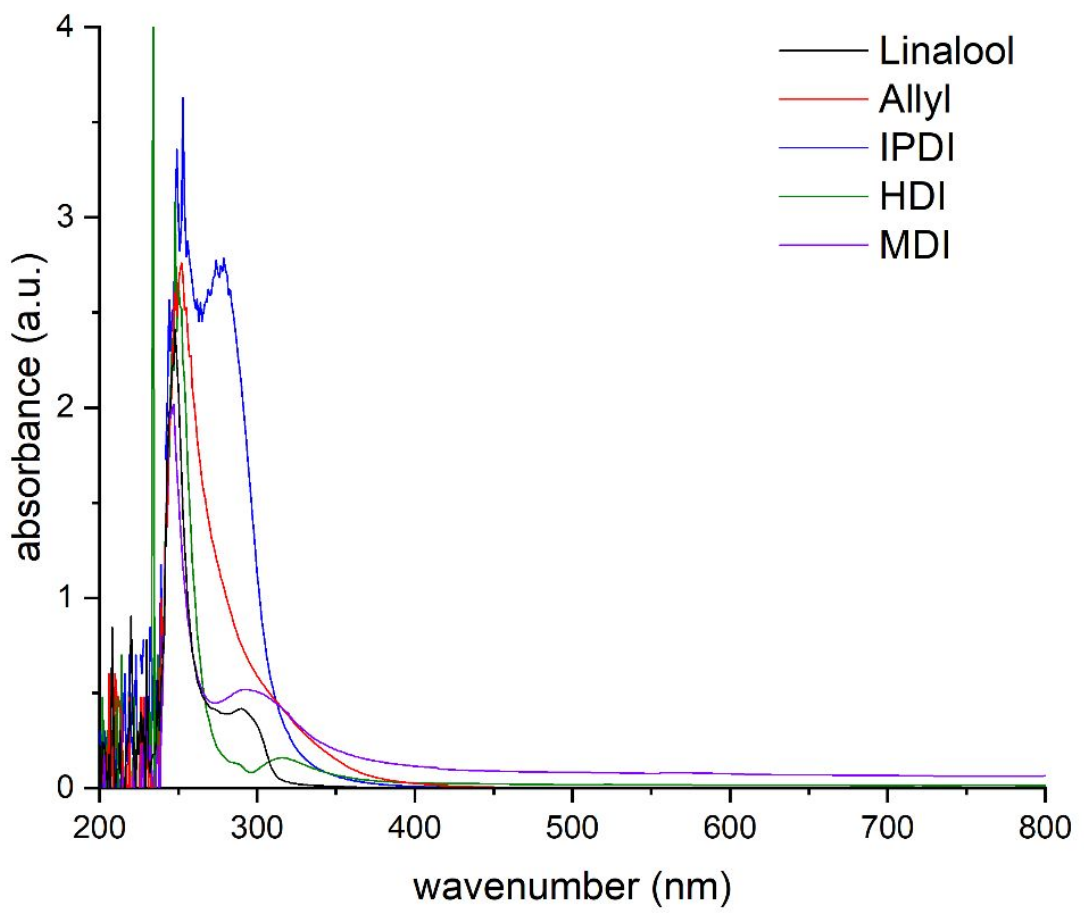

Figure S13. Representative UV-vis spectra curves for linalool derivative monomers tested in spectroscopic grade acetone. $(n=3)$ 
Thermomechanical Characterization

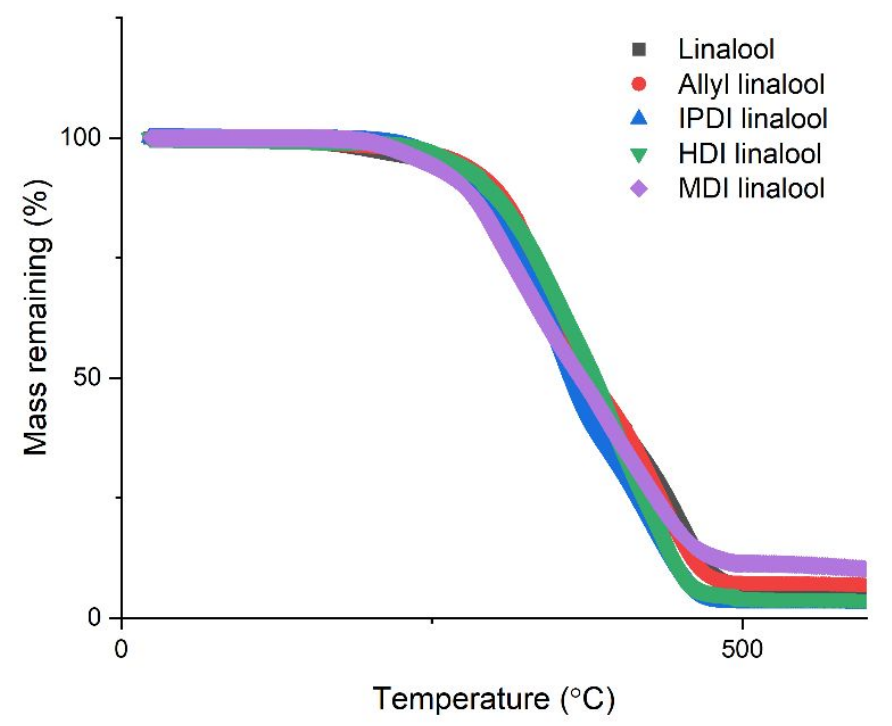

Figure S14. Representative TGA curves for linalool derivative thiol-ene thermoset films tested at $10{ }^{\circ} \mathrm{C} \times$ $\min ^{-1}$ in a nitrogen atmosphere. $(n=3)$ 
A

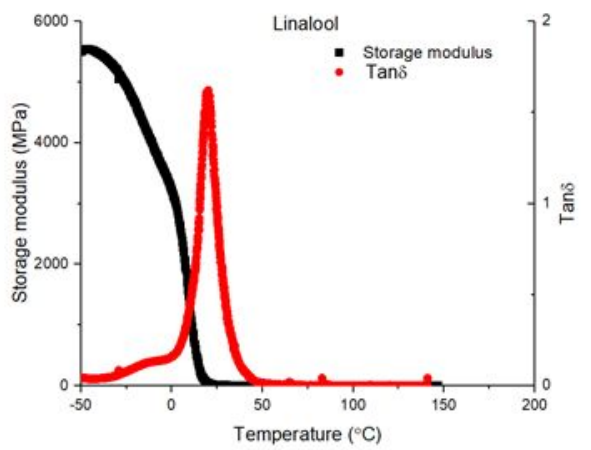

$\mathrm{C}$

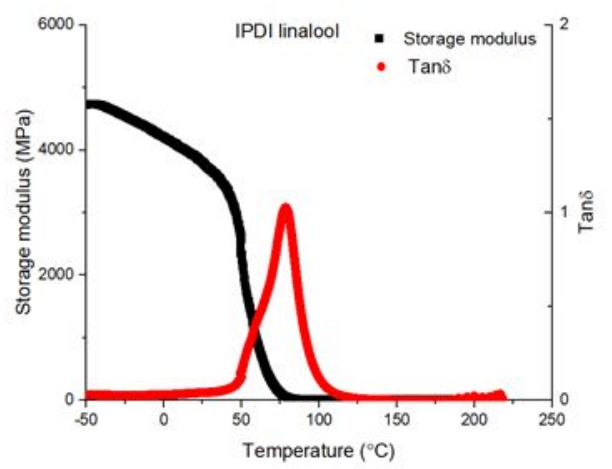

$\mathrm{E}$

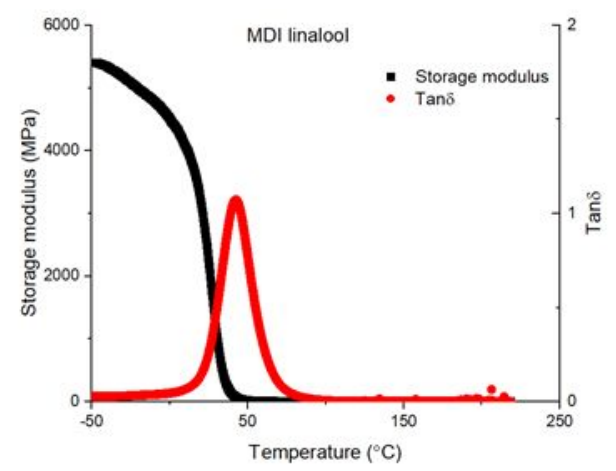

B

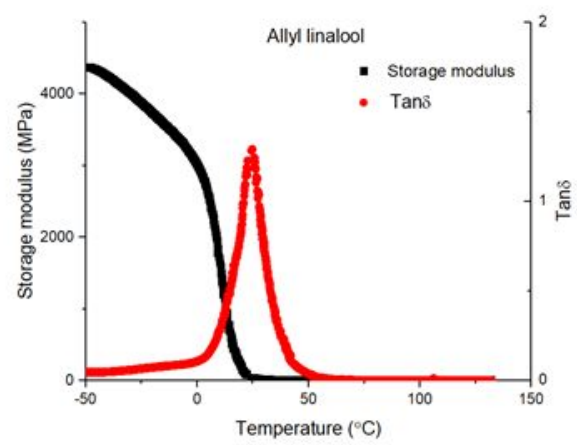

D

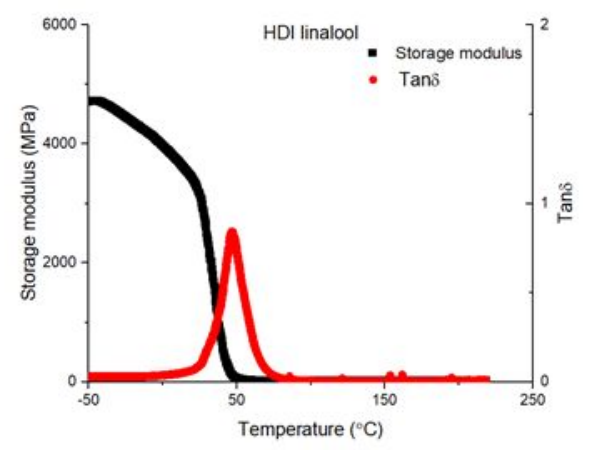

Figure S15. Representative DMA plots (storage moduli and tan $\delta$ ) for linalool (A) and linalool derivative thermosets allyl linalool (B), IPDI linalool (C), HDI linalool (D), and MDI linalool (E) tested using films at $2{ }^{\circ} \mathrm{C} \times \min ^{-1}$ from $-50{ }^{\circ} \mathrm{C}$ to $200{ }^{\circ} \mathrm{C}$. $(\mathrm{n}=3)$ 
Table S1. Mechanical properties of modified ASTM Type IV dogbones, 3D printed from linalool and linalool derived monomers. Samples were tested at $10 \mathrm{~mm} \times \mathrm{min}^{-1}$, ambient conditions ( $\left.\mathrm{n}=7\right)$.

\begin{tabular}{|l|l|l|l|l|}
\hline & Toughness (MPa) & $\begin{array}{l}\text { Strain at } \\
\text { Break (\%) }\end{array}$ & Elastic Modulus (MPa/m $\left.\mathbf{m}^{\mathbf{2}}\right)$ & Yield Strength (MPa) \\
\hline Linalool & $57.5 \pm 18.2$ & $118.2 \pm 24.4$ & $0.3 \pm 0.3$ & $4.4 \pm 1.5$ \\
\hline Allyl linalool & $46.8 \pm 13.9$ & $84.2 \pm 14.0$ & $1.3 \pm 0.7$ & $0.8 \pm 0.1$ \\
\hline IPDI linalool & $291.7 \pm 113.2$ & $16.50 \pm 18.8$ & $125.0 \pm 34.4$ & $15.7 \pm 7.4$ \\
\hline HDI linalool & $673.7 \pm 96.7$ & $70.3 \pm 10.0$ & $77.5 \pm 12.7$ & $9.6 \pm 1.2$ \\
\hline MDI linalool & $329.3 \pm 81.3$ & $112.7 \pm 14.9$ & $12.6 \pm 1.9$ & $3.0 \pm 1.2$ \\
\hline
\end{tabular}



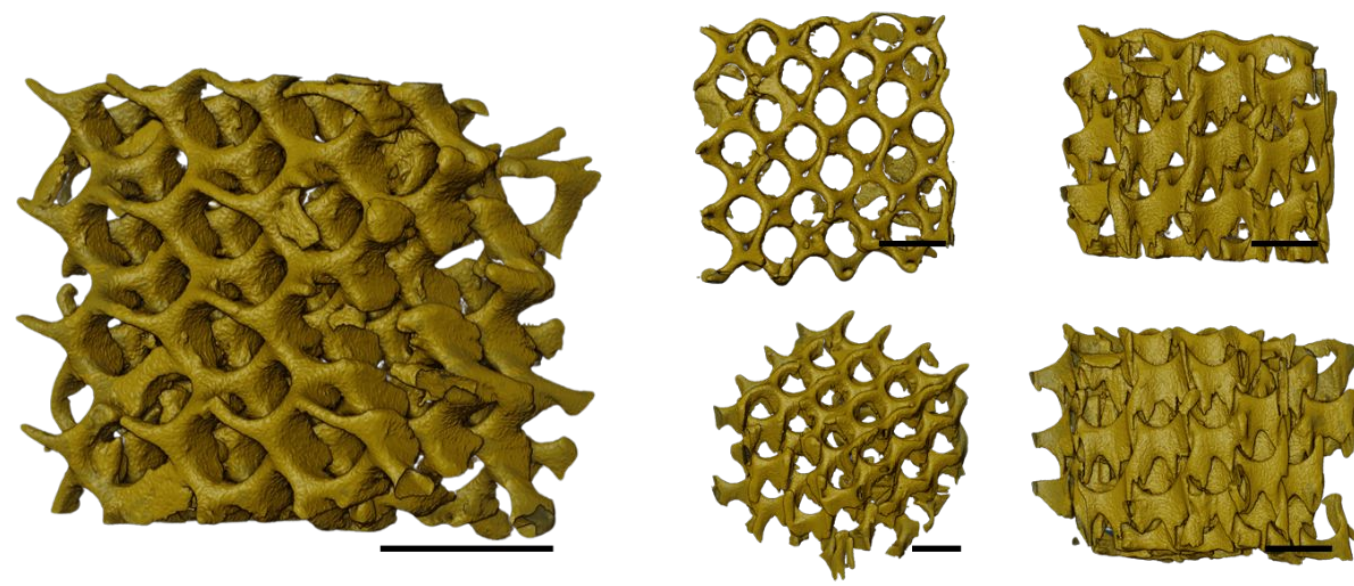

Figure S16. Reassembled micro-CT images of an HDI scaffold, displaying different views including possible overcuring $($ scale bar $=0.5 \mathrm{~cm})$ 

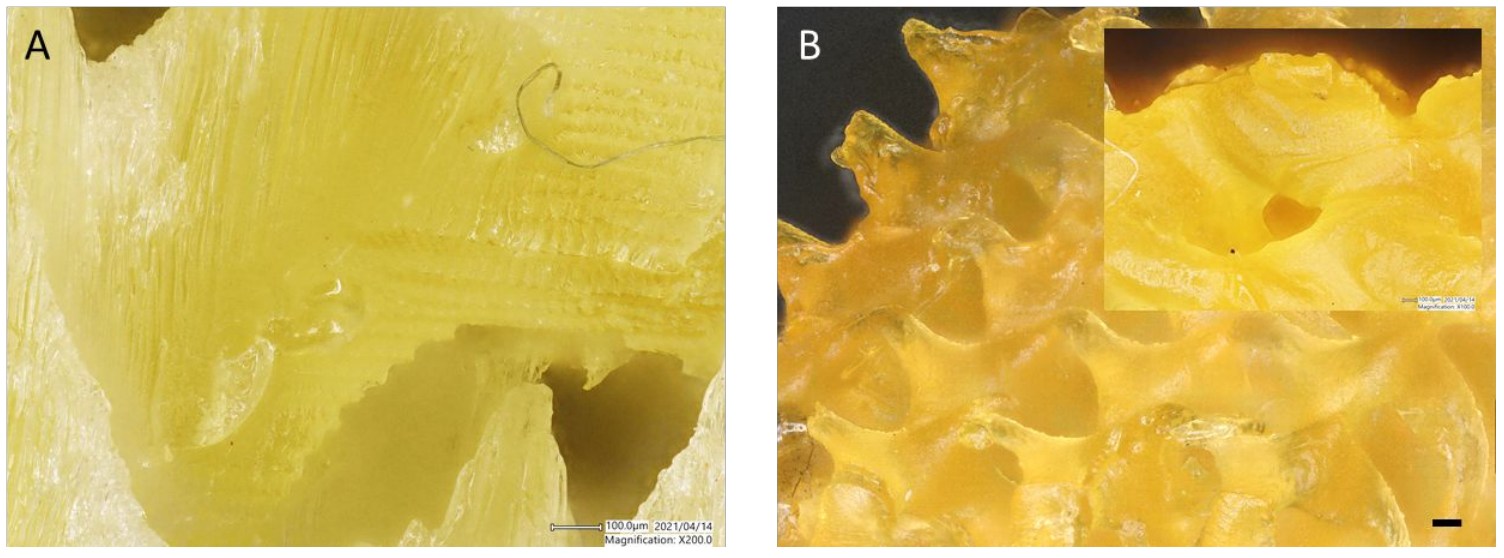

Figure S17. Light micrographs of HDI linalool scaffold (A) and MDI linalool scaffold (B) (scale bar $=0.1$ $\mathrm{cm})$ 

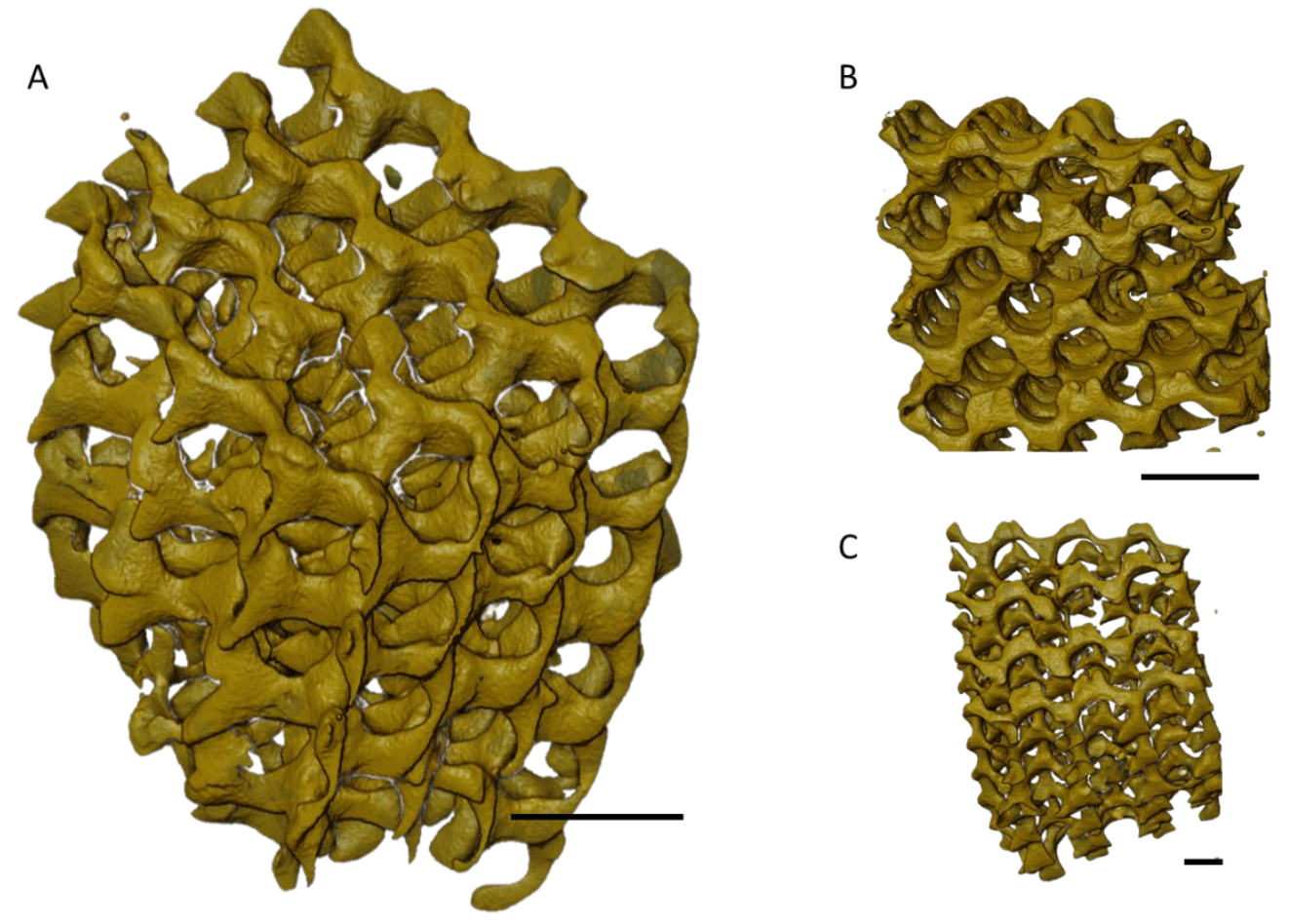

C

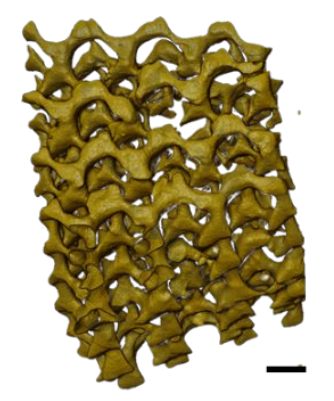

Figure S18. Reassembled micro-CT images of representative MDI linalool porous scaffolds (A-C) (scale bar $=0.5 \mathrm{~cm})$ 


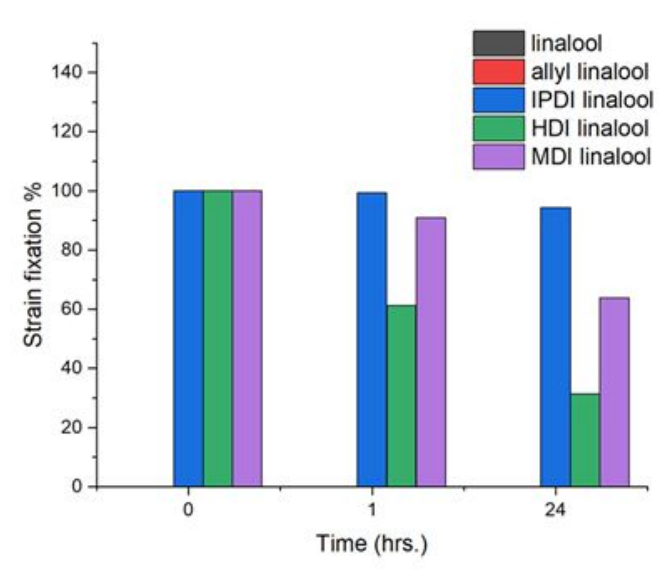

Figure S19. 4D behavior of the linalool-derived thermosets, including strain fixation at ambient conditions. The control linalool (no modification) and allyl linalool are included but displayed no strain fixation at room temperature. $(\mathrm{n}=3)$ 